\title{
Reel-to-Reel Electron Microscopy: Latency-Free Continuous Imaging of Large Sample Volumes
}

Christopher S. Own ${ }^{1}$, Matthew F. Murfitt ${ }^{1}$, Lawrence S. Own ${ }^{1}$, Derrick Brittain ${ }^{2}$, Nuno da Costa ${ }^{2}$, R. Clay Reid ${ }^{2}$, David G. C. Hildebrand $^{3}$, Brett Graham ${ }^{3}$, \& Wei-Chung Allen Lee ${ }^{3}$.

${ }^{1}$ Voxa, Seattle, WA USA

${ }^{2}$ Allen Institute for Brain Sciences, Neural Coding group, Seattle, WA USA

${ }^{3}$ Harvard Medical School, Department of Neurobiology, Boston, MA USA

The electron microscope (EM) provides exquisitely detailed information about structural arrangements of matter through its high native resolution, contrast and wide variety of available signals. This enables broad application across numerous fields, including physics, materials science, medicine, and biology. In some fields, especially biology, there is increasing need for quantification at smaller length scales and simultaneous demand for structural data from larger volumes. While new digital automated tools make it possible in some cases to investigate most of a $3 \mathrm{~mm}$ transmission EM sample, they remain ineffective for significantly larger volumes, for example whole-genome patterned DNA [1], neural circuits [2], silicon wafers, and histological arrays. This is due in large part to operating cost measured in both dollars and hours - arising from extensive sample preparation / handling and dependence on skilled operators [3]. For this reason, applicability of high-resolution EM beyond laboratory research is mostly limited to niche areas (for example, nephrology and ciliary dyskinesia in clinical medicine). Further, researchers and clinicians increasingly turn to methods that take ensemble measurements such as low-cost genetic sequencing and mass spectrometry despite the richness and spatial precision of information available from EM.

Published EM results are generally cherry-picked from dozens to hundreds of images of painstakingly-prepared samples taken over weeks to months. What if EM's were optimized such that every image coming from the machine was scientifically significant and "publication-ready"? High throughput requires new ways of looking at EM imaging. The EM imaging process is a packet system, and throughput can be defined as the amount of useful data retrieved from the system during the packet divided by the time taken to do so. To be an honest throughput number, the time taken should include all aspects of the experiment, including sample preparation and loading, machine setup time (and any downtime experienced), as well as the time spent in the microscope itself. Generally the time spent actually examining the sample in the microscope is tiny compared to these other steps, and the time spent acquiring scientifically significant data is an even smaller fraction.

Microscopy needs to significantly change to meet the throughput needs of modern biology: high resolution imaging may transition from a seldom-used relatively small part of a lengthy process to an always-on, always available part of a long term, continuous acquisition chain lasting weeks, months or even years. In this paradigm, collecting scientifically significant images becomes the majority of the process, rather than a small part of an arduous march dominated by sample preparation, sample handling, and experiment design. For a microscope to operate efficiently in this paradigm, it needs to offer robust and reliable performance over very long timescales. Such automation has begun with scanning EM [4] and work in EM-based gene sequencing [5,6]. Transmitted electron detection, however, has the distinct advantages of higher resolution, greater signal to noise, and is faster as area detectors are readily parallelizable. Throughput would then not be measured in megapixels per second, but rather terapixels per week, or even petapixels per year. The microscope needs native advanced, intelligent, and pre-emptive diagnostics able to provide notification of any problems or service required, if possible before they even occur. Once microscopes reach this level of reliability and sustainability, it 
is likely that other problem domains will start to find applications for such a service.

At Voxa, we have been producing new tools to provide solutions of this type, and report here on a novel tape-based in-column, reel-to-reel sample handling system for EM imaging produced by Voxa called GridStage $^{\mathrm{TM}}$ (Fig. 1). Voxa's GridStage ${ }^{\mathrm{TM}}$ is derived from high-throughput pipeline elements developed by Voxa, and enables high-throughput EM imaging with maximum reproducibility. This pipeline was previously used to enhance the effective throughput on Hitachi and Nion stock instruments by 4-5 orders of magnitude in a novel EM-based DNA sequencer [5]. GridStage ${ }^{\mathrm{TM}}$ is compatible with a variety of tape-based sample carriers such as the chip-carrier tape shown in Fig. 2, with capacity exceeding 10,000 samples per reel, and will be transformative for applications that demand reduced sample handling, large volumes with high spatial information density, and consistency from sample preparation through to analysis.

GridStages ${ }^{\mathrm{TM}}$ are currently retrofitted to $120 \mathrm{kV}$ JEOL 1200EX microscopes augmented with custom high-speed TEM camera arrays (TEMCAs) developed by coauthors at Harvard Medical School [2], and together are being used to acquire massive maps of neural circuits from serial sections of neural tissue. Previously, the prospects for mapping mammalian brain connectivity at the synaptic level spanned thousands to tens of thousands of years with conventional tools, an impractical endeavor. GridStage $^{\mathrm{TM}}$ combined with TEMCA enables consistent high-speed automated operation with a theoretical bandwidth exceeding 100 megapixels per second, and advancement between samples in seconds. Image acquisition, sample rastering, and sample advance are completely automated and optimized; the system is accessed remotely via the web through a lightweight Python-based programming interface, enabling full monitoring of acquisition progress and datastreams from unlimited users. With GridStage ${ }^{\mathrm{TM}}$ and other throughput-enhancing tools by us and others [7], whole brain synaptic-resolution structural maps could potentially be in reach within a decade [8].

\section{References:}

[1] Payne AC, et al, PLoS ONE 8 (2013), 1-11.

[2] Bock D, et al, Nature 471 (2011), 177-182.

[3] Peddle C \& Collinson L, Micron 61 (2014), 9-19.

[4] Denk W \& Horstmann H, PLoS Biology 2 (2004), 1900-1909.

[5] Own C, et al, Microscopy \& Microanalysis 19 (2013), 208-209.

[6] Bell DC, et al, Microscopy \& Microanalysis 18 (2012), 1049-1053.

[7] Marx V, Nature 503 (2013), 147-152.

[8] This work was supported by Voxa, NIH grant R21 NS085320, and through resources provided by the Allen Institute for Brain Science. Drs. W. Ho and K. Hayworth are thanked for many useful discussions and contributions to this work.

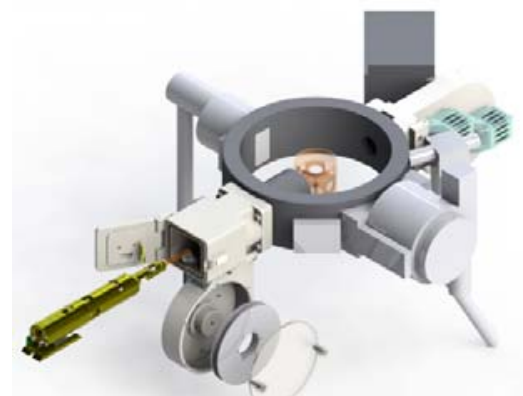

Figure 1. Section view of GridStage ${ }^{\mathrm{TM}}$ reel-to-reel sample handing system and high speed rastering cartridge installed on JEOL 1200EX microscope column. The exploded view shows a reel. Tape advances from the feed reel (left) to the takeup reel (right).

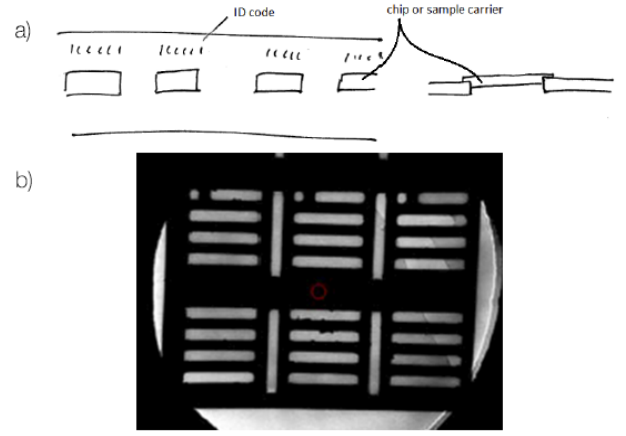

Figure 2. a) Example tape used to carry microfabricated sample support chips, b) section of microfabricated sample support chip featuring $<5 \mathrm{~nm}$ thick polymer support films suspended over 2 × 20 um slots, designed for linear polymer imaging. 